\title{
CARACTERIZAÇÃO DE NANOFLUIDOS PARA UTILIZAÇÃO EM COMPRESSORES DE REFRIGERAÇÃO: DETERMINAÇÃO DA VISCOSIDADE DINÂMICA, CONDUTIVIDADE TÉRMICA E ESTABILIDADE
}

\author{
C. R. F.BARBOSA ${ }^{1}$, E. H. V. SOUSA ${ }^{2}$, F. A. O. FONTES ${ }^{3}$, I. M. G. ALMEIDA ${ }^{4}$, V. F. AZEVEDO ${ }^{5 *}$ \\ ${ }^{1,4}$ Universidade Federal do Rio grande do Norte \\ ${ }^{2}$ Instituto Federal de Educação, Ciência e Tecnologia deAlagoas \\ ${ }^{3,5^{*}}$ Instituto Federal de Educação, Ciência e Tecnologia do Rio grande do Norte \\ valerio.azevedo@ifrn.edu.br
}

Artigo submetido em 04/12/2015 e aceito em 29/11/2016

DOI: $10.15628 /$ holos.2016.3802

\section{RESUMO}

Este trabalho identifica a viscosidade dinâmica e condutividade térmica de três nanofluidos (NFs) a base de óleo mineral ISO 32, utilizado em compressores de refrigeração, aditivados respectivamente por nanopartículas (NPs) de nanotubos de carbono (NTC), óxido de nióbio $\left(\mathrm{Nb}_{2} \mathrm{O}_{5}\right)$ e Índio (In). Após os ensaios termofísicos, verifica-se a estabilidade dos (NFs) através de métodos específicos e em seguida, compara-se o desempenho tribológico da amostra mais estável com o fluido base puro através de ensaio de lubricidade em bancada de alta frequência. Os resultados demonstraram que a viscosidade dinâmica tem alteração desprezível, a condutividade térmica tem significativo ganho em relação ao óleo puro e que os (NFs) de (NTC) e (In), possuem boa e razoável estabilidade respectivamente, enquanto que os compostos aditivados com (NPs) de $\left(\mathrm{Nb}_{2} \mathrm{O}_{5}\right)$ são instáveis e não são viáveis para a aplicação em sistemas térmicos. Os NFs de (NTC) ao serem comparados com o óleo puro, apresentaram maior espessura do filme lubrificante, menor coeficiente de atrito e menor desgaste médio.

PALAVRAS-CHAVE: nanofluidos, nanopartículas, viscosidade dinâmica, condutividade térmica e estabilidade.

\section{CHARACTERIZATION OF NANOFLUIDS FOR USE IN REFRIGERATION COMPRESSORS: DETERMINATION OF DYNAMIC VISCOSITY, THERMAL CONDUCTIVITY AND STABILITY}

\begin{abstract}
This paper identifies the dynamic viscosity and the thermal conductivity three nanofluids (NFs) mineral oil base ISO 32, used in domestic refrigeration compressors, additivated respectively by nanoparticles (NPs) of carbon nanotubes (NTC), niobium oxide $\left(\mathrm{Nb}_{2} \mathrm{O}_{5}\right)$ and Indian (In).After analyzing the thermo-physical properties, investigates the stability (NFs) by specific methods and then is compared the tribological performance of the most stable sample by high frequency reciprocating rig. The results demonstrated
\end{abstract}

that the dynamic viscosity has insignificant change, the thermal conductivity has significant gain with respect to the pure oil and that (NFs) of (NTC) and (In) have good and reasonable stability respectively, while the compounds additivated with (NPs) of $\left(\mathrm{Nb}_{2} \mathrm{O}_{5}\right)$ are unstable and are not viable for use in thermal systems. The (NFs) of(NTC) when compared with the pure oil showed higher lubricant film thickness, lower coefficient of friction and lower average wear.

KEYWORDS: nanofluids, nanoparticles, dynamic viscosity, thermal conductivity and stability. 


\section{INTRODUÇÃO}

Fluidos convencionais utilizados na refrigeração desempenham um importante papel em sistemas térmicos, porém, a necessidade de compactação dos equipamentos despertou o interesse por soluções que aprimorem a transferência de calor, sem comprometer o desempenho dinâmico destes sistemas.

Assim, foram iniciados estudos sobre a adição de partículas sólidas aos fluidos bases, a princípio em microescala e observou-se que os compostos gerados contribuíam na melhoria do desempenho das propriedades térmicas, porém geravam problemas como entupimentos de capilares, aumento do atrito e perda de carga. Posteriormente, os esforços se concentraram na adição de partículas nanométricas, menores que as anteriores em três ordens de grandeza.

As investigações sobre o comportamento térmico dos nanofluidos (NFs) convergem para quatro seguimentos distintos, sendo eles: melhoria da condutividade térmica, transferência de calor por convecção, transferência de calor com mudança de fase e redução do desgaste (atrito).

A adição de nanopartículas (NPs) ao fluido base tem a tendência predominante de aumentar a viscosidade dinâmica do composto, mas esse resultado sofre considerável influência da temperatura, onde seu valor tem expressiva redução para temperaturas elevadas. 0 conhecimento da viscosidade do composto é fundamental para o estudo do efeito tribológico, para a taxa de transferência de calor e também para a queda de pressão nos sistemas térmicos.

A estabilidade é um importante fator para a comprovação da viabilidade dos (NFs). Além de prejuízos relacionados com a mensuração dos resultados, esta característica é muito importante para a determinação da intensidade térmica do composto. Assim, frequentemente recorre-se a adição de dispersantes que tem como finalidade dificultar a sedimentação das (NPs).

Em sistemas de refrigeração, componentes mecânicos do compressor possuem movimentos relativos que geram incrementos nos níveis de atrito, implicando na perda de material por desgaste. Estudos procuram meios de lubrificação que reduzam o contato direto entre essas peças e consequentemente aumentem a confiabilidade e durabilidade destes equipamentos.

Melhorias significativas nos resultados da condutividade térmica em (NFs) à base de óleos são adquiridos ao se utilizar (NPs) metálicas de cobre (Cu), alumínio (Al), índio (In) e prata (Ag), mas outros materiais não metálicos também apresentam consideráveis desempenhos nesta utilização, destacando-se principalmente, (NPs) de óxido de alumínio $\left(\mathrm{Al}_{2} \mathrm{O}_{3}\right)$, óxido de nióbio $\left(\mathrm{Nb}_{2} \mathrm{O}_{5}\right)$ e nanotubos de carbono (NTC).

Segundo Pastoriza-Gallego, Casanova, Legido e Pioneiro (2011), a forma e a estrutura sólida das (NPs) são importantes para o aumento da condutividade térmica. Esses autores relatam que obtiveram melhores resultados ao utilizarem (NPs) com superfícies alongadas.

Para Yu, France, Routbord e Choi (2008) um (NF) não é apenas uma simples mistura líquido-sólido. A sua síntese deve reunir condições como: suspensões uniformes, estáveis e duráveis e com baixa aglomeração de partículas, sendo utilizados processos físicos e químicos para a sua obtenção. Eles observaram alguns compostos que apresentaram aumento de transferência de calor na faixa de 15 e $40 \%$. 
Duanghthongsuk e Wongwises (2009) descobriram que (NFs) com (NPs) de $\left(\mathrm{Al}_{2} \mathrm{O}_{3}\right)$ em água têm melhor condutividade térmica quando concentra-se maior número de partículas, porém esta propriedade é ligeiramente reduzida com o aumento da temperatura.

Chen, Ding, He e Tan (2007) observaram um comportamento newtoniano em (NFs) de óxido de titânio $\left(\mathrm{TiO}_{2}\right)$ em etilenoglicol. Este mesmo tipo de comportamento também foi observado em um (NF) de $\left(\mathrm{Al}_{2} \mathrm{O}_{3}\right)$ em água por Chandrasekar, Suresh e Chandra (2010).

Lotfizadeh e Matsoukas (2015) apresentaram o seguinte modelo teórico para a determinação da condutividade térmica de uma mistura de dois componentes quando a razão entre a condutividade das duas fases é superior a 100:

$$
\mathrm{K}_{e f f}=\mathrm{K}_{f} \frac{\mathrm{K}_{p}+(\mathrm{n}-1) \mathrm{K}_{f}-(\mathrm{n}-1) \emptyset\left(\mathrm{K}_{f}-\mathrm{K}_{p}\right)}{\mathrm{K}_{p}+(\mathrm{n}-1) \mathrm{K}_{f}+\emptyset\left(\mathrm{K}_{f}-\mathrm{K}_{p}\right)}
$$

Nesta expressão, $\mathbf{K}_{\text {eff, }}, \mathbf{K}_{p}$ e $\mathbf{K}_{f}$, representam a condutividade térmica do (NF), da (NP) e do fluido base respectivamente, enquanto que $\varnothing$ representa a fração volumétrica e $\mathbf{n}$ o fator de forma da partícula, que é definido por $n=3 / \psi$, sendo $\psi$ a razão entre a área superficial de uma esfera (com o mesmo volume que o da partícula) e a área superficial da partícula, ou seja, a esfericidade da partícula.

Salinas (2005) comenta que os modelos propostos para estimar a viscosidade dos (NFs) são derivados do modelo de Einstein que utilizou as equações de Navier-Stokes, para obter a viscosidade efetiva de um fluido em um escoamento incompressível. Neste modelo as partículas são esferas rígidas $\left(\boldsymbol{\theta}_{v}\right)$, sem interações, e bem maiores do que as moléculas do solvente. Neste modelo a viscosidade do (NF) e a do fluido base, são representadas respectivamente por $\left(\boldsymbol{\mu}_{\boldsymbol{n} \boldsymbol{f}}\right)$ e $\left(\boldsymbol{\mu}_{f}\right)$ se relacionam através de:

$$
\mu_{\mathrm{nf}}=\left(1+2,5 \theta_{v}\right) \mu_{f}
$$

Timofeeva, Yu, France, Singh e Routbort (2011) comentam que normalmente a viscosidade aumenta à medida que o tamanho das partículas diminui e quando a fração de volume aumenta, mesmo se, por vezes, um comportamento diferente é observado.

Zhu et al. (2009) estudaram um (NF) de $\left(\mathrm{Al}_{2} \mathrm{O}_{3}\right)$ e água, e concluíram que sua condutividade térmica pode ser melhorada com a adição do tensoativo dodecilbenzeno sulfato de sódio (SDBS) como dispersante em uma quantidade e valor de $\mathrm{pH}$ controlada.

Yang, Zhang, Grulke, Anderson e Wu (2005) investigaram a transferência de calor por convecção de (NPs) de grafite dispersas num fluido base em fluxo laminar num trocador de calor tubular horizontal e constataram que o coeficiente de transferência de calor deste (NF) aumentou em $22 \%$ para uma adição de $2,5 \%$ de (NPs).

A obtenção de um (NF) nada mais é que a formação de um colóide que tem sua estabilidade sobre forte dependência das medidas das propriedades da fase dispersa. Assim, a proposta deste trabalho é estudar a viabilidade da utilização de nanofluidos gerados a partir de (NPs) de (In), $\left(\mathrm{Nb}_{2} \mathrm{O}_{5}\right)$ e (NTC) em óleos minerais ISO 32 utilizados em compressores de refrigeração. 


\section{MATERIAIS E MÉTODOS}

Os materiais utilizados nas composições têm as seguintes características:

- Fluido base: Óleo mineral CHAMP RF 32, constituído de mineral naftênico, especialmente tratado para a lubrificação de compressores de refrigeração de sistemas selados.

- Material de adição: (NPs) de (NTC), $\left(\mathrm{Nb}_{2} \mathrm{O}_{5}\right)$ e (In), adquiridas da SIGMA-ALDRICH. Os nanotubos possuem comprimento menor que $20 \mathrm{~nm}$ e diâmetro menor que $50 \mathrm{~nm}$, enquanto que as (NPs) de $\left(\mathrm{Nb}_{2} \mathrm{O}_{5}\right)$ e (In) têm estrutura esférica e possuem diâmetro menor que $50 \mathrm{~nm}$.

A preparação dos (NFs) foi realizada obedecendo às seguintes etapas:

I. Pesagem das (NPs) - Após calcular a quantidade da fração de (NP) que deverá ser adicionada ao óleo, realiza-se a pesagem em uma balança de precisão de 0,001 g.

II. Adição das (NPs) - Gradua-se $500 \mathrm{ml}$ do óleo ISO 32 em um becker de vidro de $600 \mathrm{ml}$ e adiciona-se as (NPs), a seguir com o auxílio de uma vareta de vidro, mistura-se manualmente a composição.

III. Desfragmentação - Coloca-se o composto bifásico em banho ultrassônico, que produz alta intensidade de energia acústica no processo homogeneização de materiais com a finalidade de causar permanente mudança física e química devido a geração de cavitação e micro fluxos nos líquidos, aquecimento e ruptura nos sólidos, reduzindo dessa forma possíveis aglomerações de (NPs). Recomenda-se que este ensaio seja realizado em temperatura ambiente por um período de 24 horas.

IV. Homogeneização - Para finalizar a fase de preparação, utiliza-se um equipamento destinado a agitar a solução por meio de uma pequena barra magnética movida por um campo rotativo, que acelera a movimentação das composições. Recomenda-se que os (NFs) sejam agitadas por $24 \mathrm{~h}$ a 450 RPM.

A figura 1 apresenta o aspecto visual dos (NFs) preparados para as análises experimentais.

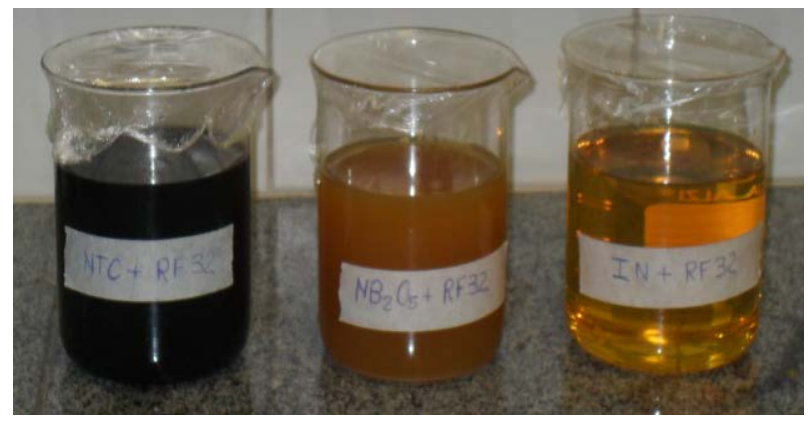

Figura 1 - Aparência visual dos (NFs) após a preparação. 


\section{METODOLOGIA DOS ENSAIOS}

Os resultados que embasaram a análise deste trabalho são oriundos dos seguintes procedimentos experimentais:

- Viscosidade Dinâmica - Ensaio realizado através de um reômetro tipo Brookfield Digital Rheometer Model DV-III+ banho termostático TC-550, interligado a um PC. Este experimento ocorreu nas três amostras preparadas e no óleo ISO $32 \mathrm{sem}$ mistura para fins de comparações futuras. As medições aconteceram no intervalo entre $20^{\circ} \mathrm{C}$ a $60^{\circ} \mathrm{C}$ com incrementos de $5^{\circ} \mathrm{C}$ e uma rotação fixada em 20 RPM. Cada medição foi repetida três vezes, com o intuito de garantir a repetitividade assumindo-se seu valor médio. A figura 2 apresenta o reômetro e o banho termostático utilizado nos ensaios.

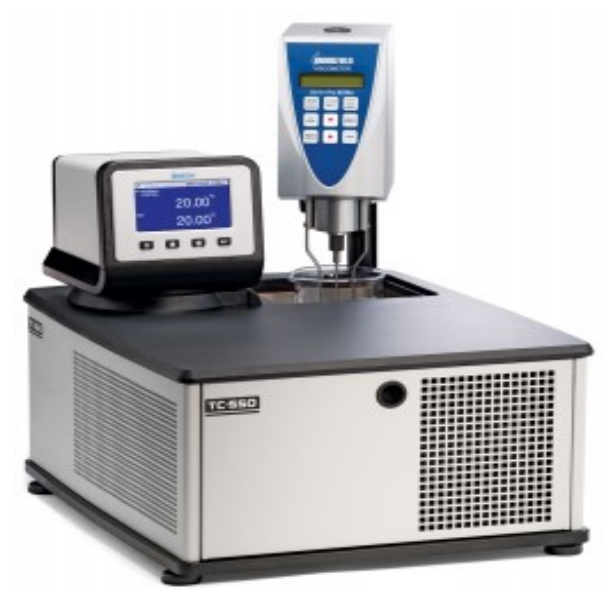

Figura 2 - Banho termostático e reômetro.

- Condutividade Térmica - Para a obtenção da condutividade térmica adicionou-se o equipamento KD2 Pro Thermal Properties Analyzer ao conjunto do ensaio anterior. O condutivímetro utilizado baseia-se no método do fio quente transiente.Com exceção da rotação que não se aplica ao ensaio, as condições e a metodologia adotada no ensaio anterior foram repetidas. A figura 3 apresenta o conjunto de equipamentos utilizados para a medição desta propriedade.

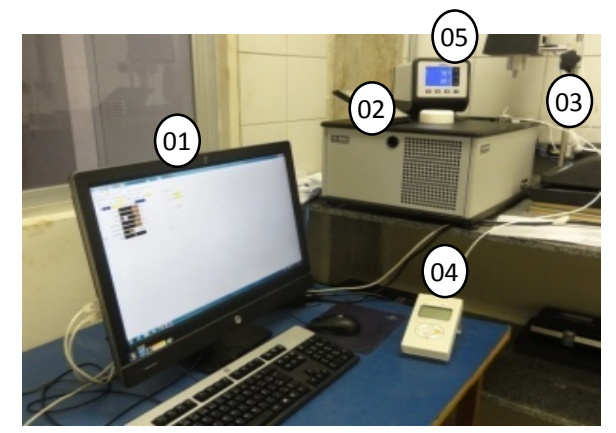

Figura 3 - Conjunto PC - 01, banho térmico - 02, reômetro - 03, condutivímetro - 04 e termopar - 05.

- Estabilidade - Após análise das propriedades térmicas, os recipientes são hermeticamente fechados e armazenados em superfície lisa à temperatura ambiente para serem monitorados periodicamente por inspeção visual para a verificação da sua estabilidade 
mecânica. A análise visual deverá ser comprovada experimentalmente através do equipamento Zeta PALS Particle Sizing.

Neste método, será mensurado o tamanho das menores e maiores aglomerações de partículas, enquanto que o nível de aglomeração é visualizado utilizando o princípio da repulsão eletrostática de partículas coloidais. A figura 4 apresenta os acessórios utilizados neste ensaio e a figura 5 o equipamento utilizado nos experimentos que determinaram o grau de aglomeração das (NPs).

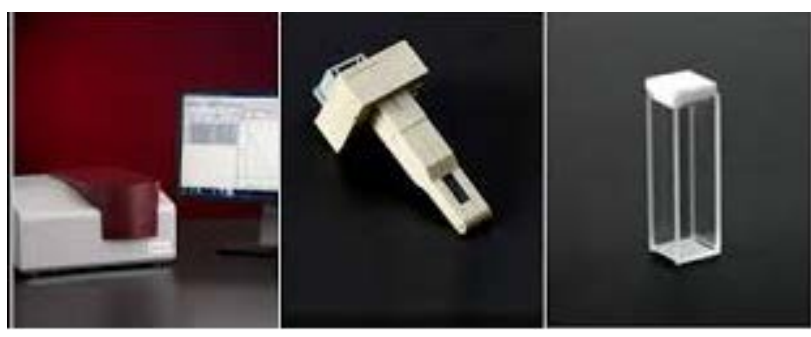

Figura 4 - Acessórios utilizados no ensaio potencial Zeta.

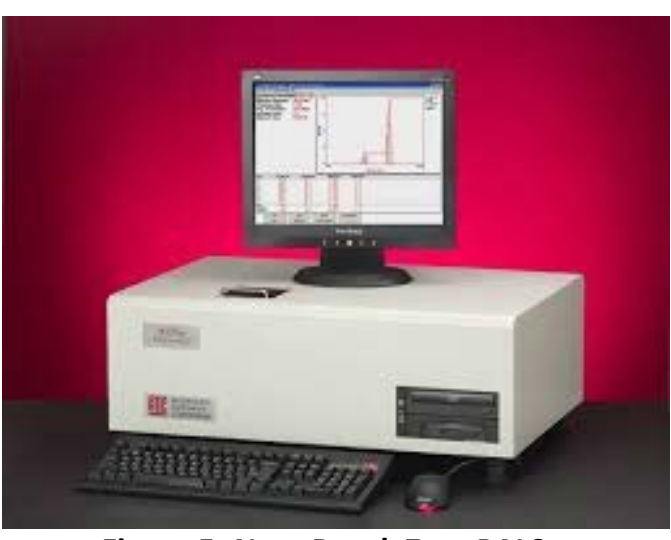

Figura 5 -NanoBrook Zeta PALS.

- Desempenho Tribológico - A amostra de melhor estabilidade será analisada em ensaio de lubricidade em bancada de alta frequência (High Frequency Reciprocating Rig - HFRR). Neste ensaio o desempenho do fluido é verificado através da espessura do filme, coeficiente de atrito e área média de desgaste do corpo de prova. O referido ensaio consiste no deslizamento de um corpo-de-prova esférico sobre uma superfície plana fixa, onde as características geométricas e físicas destes, são definidas pelas normas ASTM D 6079-99 e ISO 12156-1:1997.

O deslizamento se dá de maneira alternada com amplitudes de $1000 \mu \mathrm{m}$ e frequência de $20 \mathrm{HZ}$. No experimento, o corpo esférico e um disco plano são mergulhados em uma cuba contendo o lubrificante em análise. Para este ensaio, o conjunto foi mantido a aproximadamente $50^{\circ} \mathrm{C}$, mas a temperatura externa ao equipamento foi controlada em $25^{\circ} \mathrm{C}$. A figura 6 apresenta os equipamentos utilizados neste experimento.

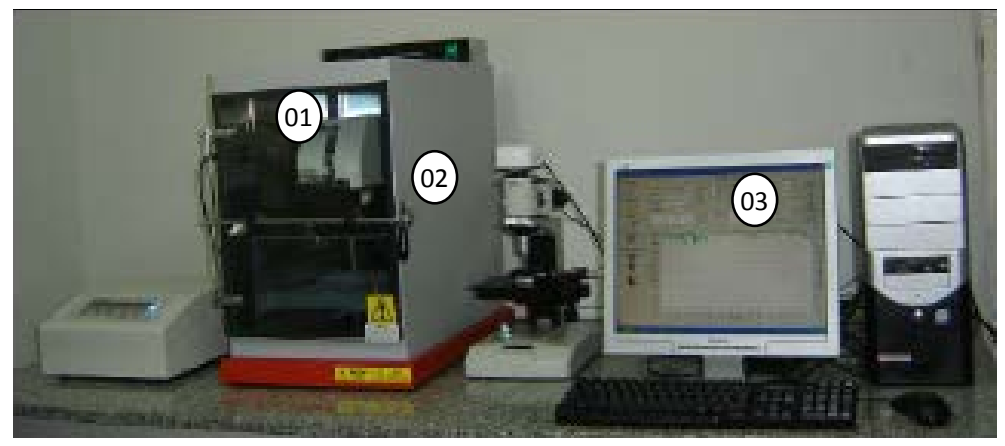

Figura 6 - Equipamento para teste de lubricidade HFRR-01, microscópio-02 e P.C.-03 


\section{RESULTADOS E DISCUSSÕES}

Viscosidade Dinâmica - A variação desta propriedade será apresentada pela figura 7. Adotou-se para todos os ensaios uma rotação de 20 RPM.

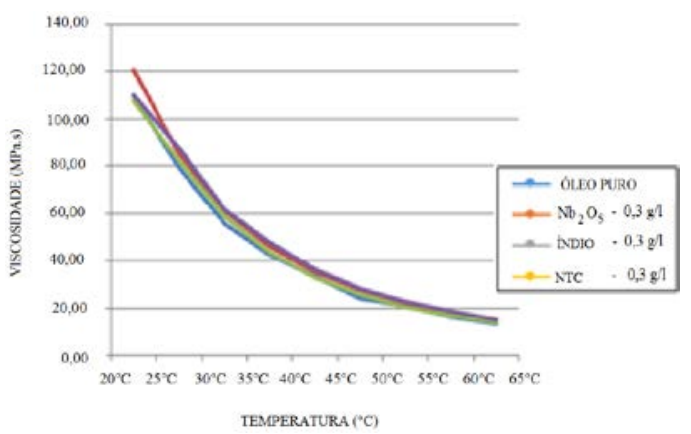

Figura 7 - Gráfico- Viscosidade (MPa.s) X Temperatura $\left({ }^{\circ} \mathrm{C}\right)$

Após a realização dos ensaios, percebe-se que a viscosidade dinâmica das amostras preparadas difere pouco desta propriedade para o óleo puro, principalmente para temperaturas acima da ambiente. Assim, consideramos esse resultado satisfatório, pois a queda de viscosidade gera problemas tribológicos nos componentes dos sistemas térmicos e uma maior viscosidade pode causar baixa eficiência nos mesmos.

Condutividade Térmica - Os valores verificados para a condutividade térmica do óleo puro e dos (NFs) de $\left(\mathrm{Nb}_{2} \mathrm{O}_{5}\right)$, (In) e (NTC) na concentração de $0,3 \mathrm{~g} / \mathrm{l}$ em função da temperatura são apresentados na figura 8.

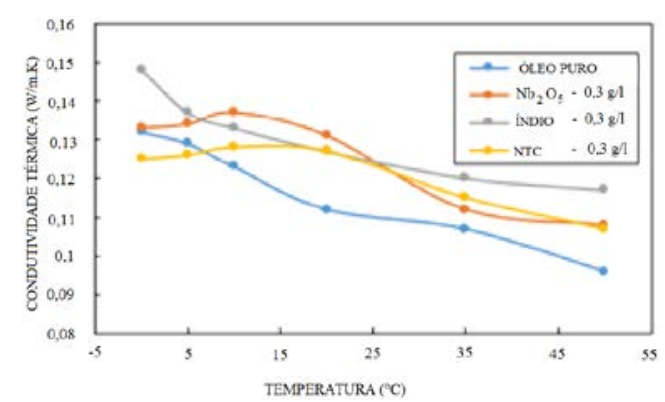

Figura8 - Gráfico- Condutividade Térmica $(\mathrm{w} / \mathrm{mK}) \times$ Temperatura $\left({ }^{\circ} \mathrm{C}\right)$

Para temperaturas menores que $7^{\circ} \mathrm{C}$ o óleo aditivado com (NPs) de (NTC) tem um desempenho menor que o óleo puro. A partir desse valor este composto começa a superar o óleo puro e demonstra uma melhor performance, principalmente a uma temperatura de aproximadamente $17^{\circ} \mathrm{C}$.

$\mathrm{O}\left(\mathrm{Nb}_{2} \mathrm{O}_{5}\right)$ demonstra melhor condutividade térmica que o óleo puro em praticamente todas as temperaturas ensaiadas, mas os resultados mais significativos estão na faixa de $7^{\circ} \mathrm{C}$ a $25^{\circ} \mathrm{C}$.

O (In) apresentou excelente performance em relação ao óleo puro. Comparando-o com os outros compostos seu desempenho só foi superado pelo comportamento do $\left(\mathrm{Nb}_{2} \mathrm{O}_{5}\right)$ na faixa de $7^{\circ} \mathrm{C}$ a $25^{\circ} \mathrm{C}$.

Estabilidade - A análise visual da estabilidade apresentou resultados positivos para os compostos de (NTC) e (In), enquanto que a amostra de $\left(\mathrm{Nb}_{2} \mathrm{O}_{5}\right)$ demonstrou considerável 
instabilidade. Assim, utilizamos o Zeta PALS Particle Sizing para analisar a comprovação do ensaio visual.

Para o (NF) composto por (NPs) de $\left(\mathrm{Nb}_{2} \mathrm{O}_{5}\right)$, o ensaio forneceu os seguintes gráficos:

(a)

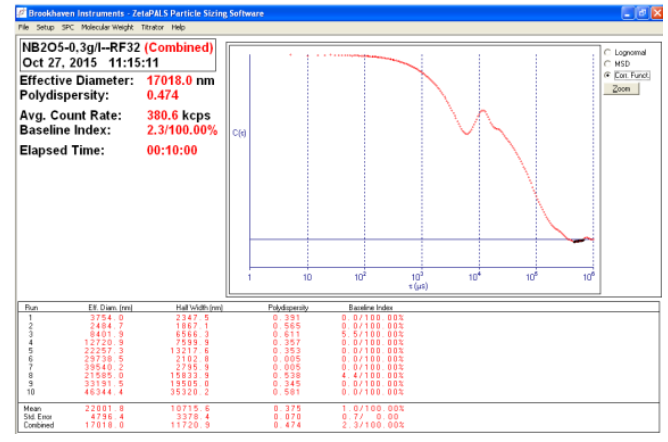

(b)

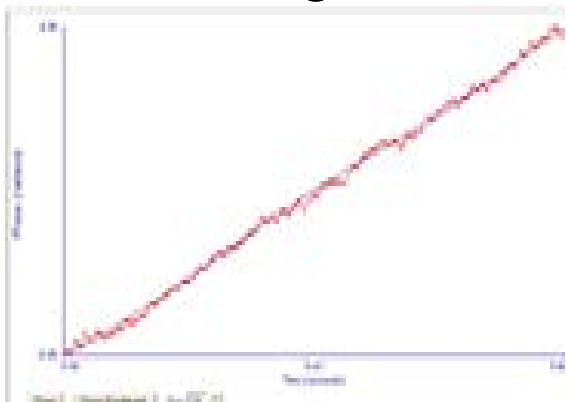

Figura9 - (a). Gráfico tamanho da aglomeração (b). Gráfico nível de aglomeração para o (NF) de $\left(\mathrm{Nb}_{2} \mathrm{O}_{5}\right)$.

O equipamento encontrou uma média de $10.716 \mathrm{nme} 22.001 \mathrm{~nm}$ respectivamente para menores e maiores aglomerações, constatando a presença de grandes aglomerações de partículas neste composto. Percebemos no gráfico da Fig. 9 (b) que as (NPs) apresentam forte atração, comprovando assim a instabilidade do (NF).

Em relação ao composto aditivado por (NPs) de (In), o ensaio forneceu os seguintes gráficos:
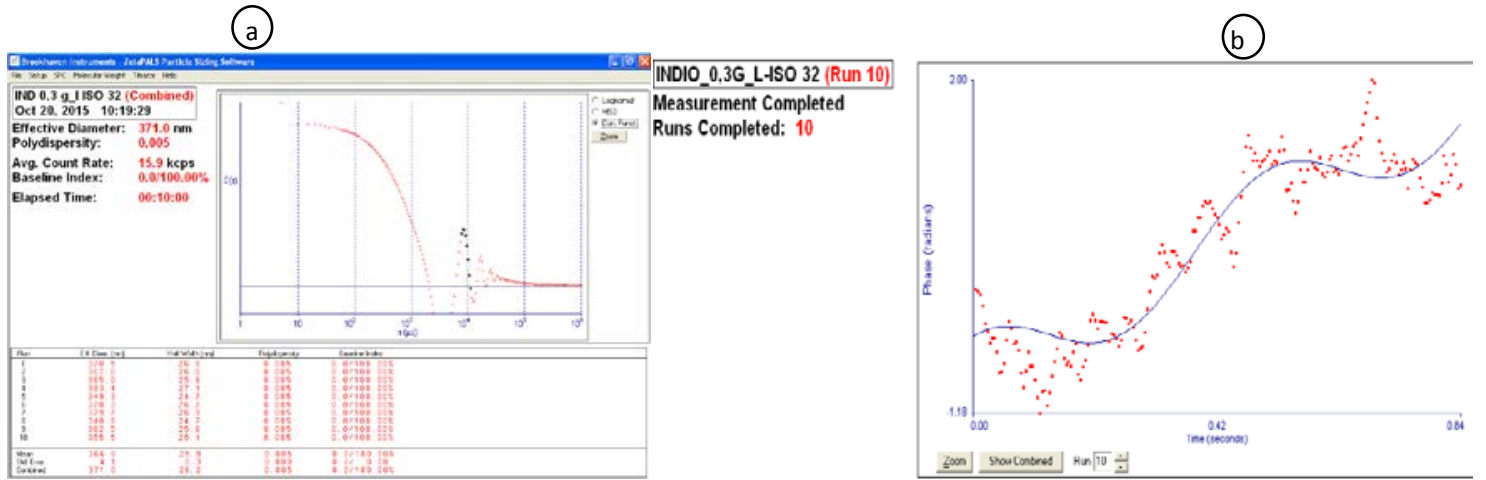

Figura10 - (a). Gráfico tamanho da aglomeração (b). Gráfico nível de aglomeração para o (NF) de (In).

O gráfico da Figura 10 (a) apresenta uma média de25,9 nm para menores aglomerações e de 366,8 nm para as maiores aglomerações encontradas. Assim, constata-se a presença de aglomerações de até 14 (NPs) neste composto. Percebemos no gráfico da Figura10 (b) que as (NPs) apresentam fraca atração, comprovando assim que o (NF) de (In) possui uma boa estabilidade.

Analogamente, para o composto aditivado por (NTC), teremos: 
(a)

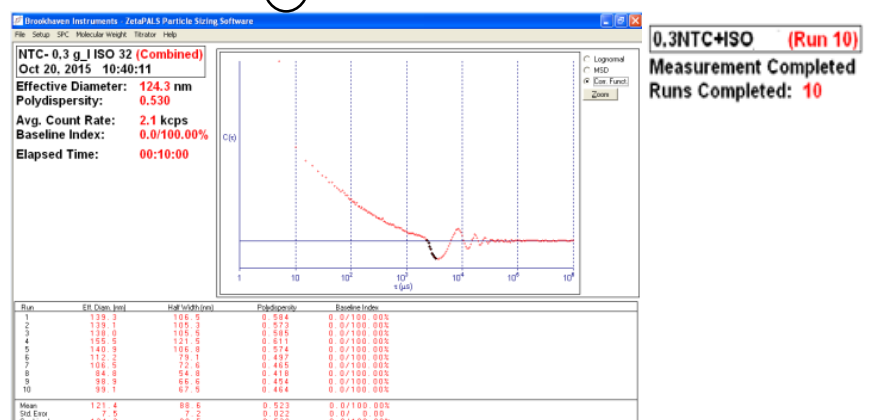

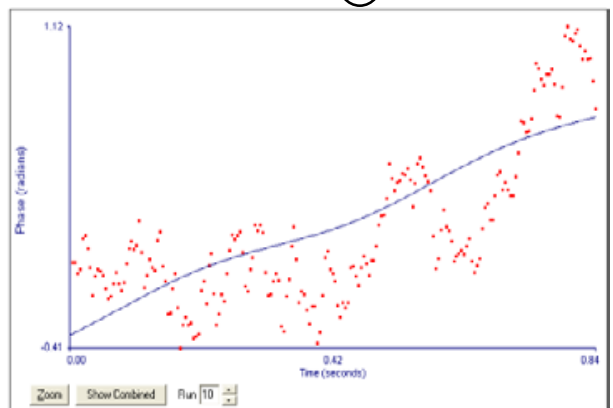

Figura 11 -(a) Gráfico tamanho da aglomeração (b) Gráfico nível de aglomeração para o (NF) de (NTC).

O gráfico da Fig. 11 (a) apresenta uma média de $88,6 \mathrm{~nm}$ para menores aglomerações e de $121,4 \mathrm{~nm}$ para as maiores aglomerações encontradas. Como estas (NPs) possuem diâmetro e comprimento, trata-se de um excelente resultado que é comprovado no gráfico da Fig. 11 (b). Assim, consideramos que entre as amostras, este composto é o de melhor estabilidade mecânica.

Desempenho Tribológico - Neste ensaio comparou-se a performance tribológica do composto formado por (NPs) de (NTC) a do óleo puro. Os parâmetros utilizados nos dois ensaios foram: Temperatura $=50^{\circ} \mathrm{C}$, Carga $=1000$ g e Frequência $=20 \mathrm{HZ}$. Cada ensaio durou 60 minutos, sendo repetido uma vez para efeitos de validação conforme normas ASTM D 6079-99 e ISO 12156-1:1997.

A Figura 12 ilustra os resultados apresentados pelas amostras de óleo ISO 32 puro e do óleo aditivado com $0,3 \mathrm{~g} / \mathrm{l}$ de (NPs) de (NTC) respectivamente.

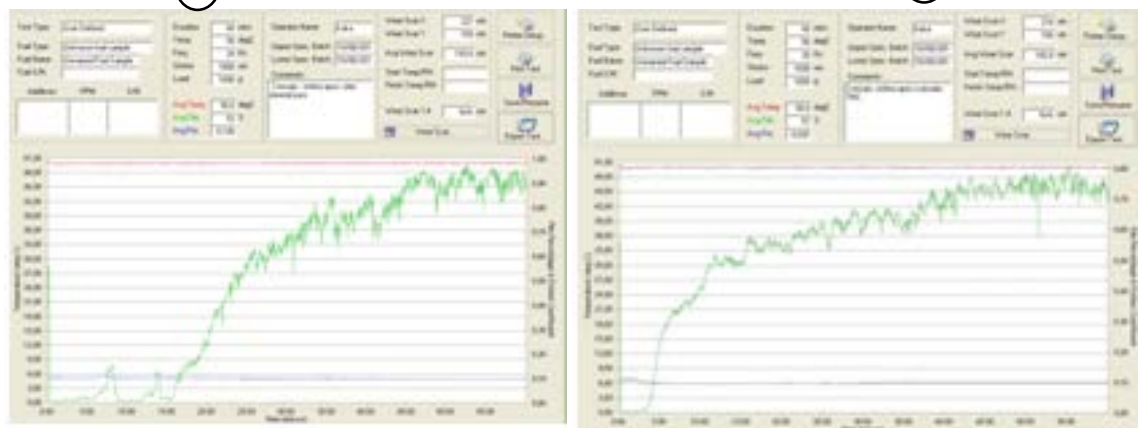

Figura 12 - Gráfico desempenho tribológico (a) Óleo ISO 32 puro (b) (NF) de (NTC) e ISO 32.

Notamos na Figura 12 que o (NF) aditivado inicia o trabalho de proteção ao desgaste bem mais rápido que lubrificante usual. Essa performance é comprovada pelos resultados obtidos para a espessura e coeficiente de atrito e desgaste nas direções, conforme ilustrado na tabela abaixo:

Tabela 1 - Parâmetros de desempenho analisados das amostras.

\begin{tabular}{lrrrrr}
\hline & $\begin{array}{c}\text { Espessura } \\
\text { do filme } \\
(\%)\end{array}$ & $\begin{array}{r}\text { Coeficient } \\
\text { e de atrito }\end{array}$ & $\begin{array}{c}\text { Desgaste } \\
\text { em } x \\
(\mu \mathrm{m})\end{array}$ & $\begin{array}{c}\text { Desgaste } \\
\text { em y } \\
(\mu \mathrm{m})\end{array}$ & $\begin{array}{c}\text { Desgaste } \\
\text { médio } \\
(\mu \mathrm{m})\end{array}$ \\
\hline Óleo mineral ISO 32 puro & 52 & 0,100 & 227 & 159 & 193 \\
Óleo mineral ISO 32 + NTC & 57 & 0,097 & 214 & 150 & 182 \\
\hline
\end{tabular}


Os corpos de prova utilizados no ensaio foram analisados em micróscopio especifco para esse experimento e apresentaram as imagens destacadas a seguir.

(a)

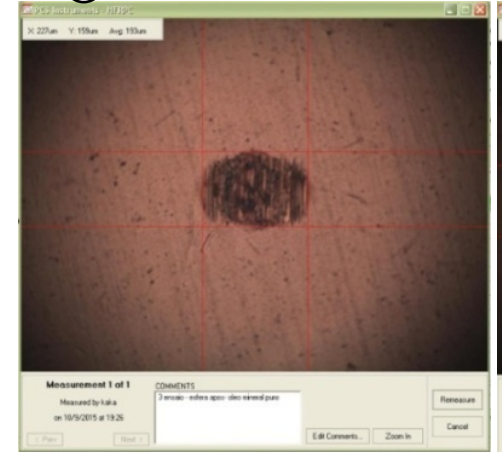

(b)

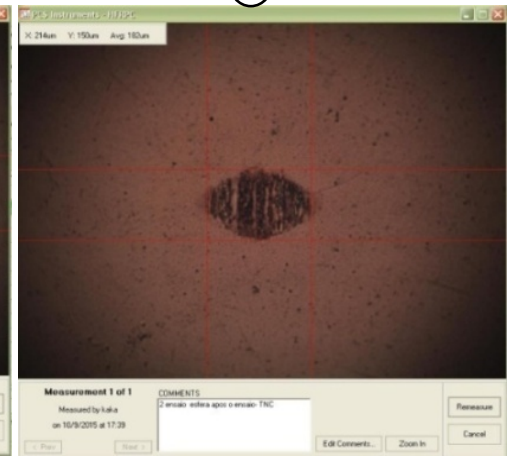

Figura 13- Exame microscópico dos corpos de prova com (a) Óleo ISO 32 puro e (b) (NF) de (NTC) e ISO 32.

Nota-se que o corpo de prova lubrificado com o óleo ISO 32 puro -(a) absorveu um desgaste maior que o corpo lubrificado com o (NF) aditivado por (NPs) de (NTC) - (b).

\section{CONCLUSÕES}

Ao adicionarmos $0,3 \mathrm{~g} / \mathrm{I}$ de (NTC) em óleo mineral ISO 68 , mantemos os mesmos valores de viscosidade dinâmica em relação ao óleo puro e percebemos tendências de melhorias na condutividade térmica do lubrificante, principalmente para temperaturas acima de 30 드, que é a faixa encontrada nos óleos utilizados em sistemas de refrigeração.

Nesta proporção o referido composto não necessita de nenhum tipo de tensoativo para manter a homogeneidade obtida na fase de preparação, além de apresentar melhor desempenho tribológico, quando comparado ao óleo ISO 32 puro.

Assim, concluímos que a adição de $0,3 \mathrm{~g} / \mathrm{l}$ de (NPs) de (NTC) em óleo mineral ISO 32, tende a melhorar a condutividade térmica e o desempenho tribológico do lubrificante em temperaturas superiores a $30^{\circ} \mathrm{C}$, não altera a viscosidade dinâmica do óleo puro e obtém significativa estabilidade.

\section{5 - REFERÊNCIAS}

1. Chandrasekar, M., Suresh, S., \& Chandra Bose, A., (2010). Experimental investigations and determination of thermal conductivity and viscosity of Al2O3/water nanofluid. Exp. Therm. Fluid Sci. 34, 210 - 216.

2. Chen, H., Ding, Y., He, Y., \& Tan, C., (2007). Rheological behavior of ethylene glycol based titania nanofluids. Chem. Phys. Lett. 444, 333 - 337.

3. Duangthongsuk, W., \& Wongwises, S., (2009). Effect of thermophysical properties models on the predicting of the convective heat transfer coefficient for low concentration nanofluid. Int. Commun. Heat Mass 35, $1320-1326$. 
4. Lotfizadeh, S., \& Matsoukas, T. (2015). Effect of nanostructure on thermal conductivity of nanofluids. Jornal of nanomaterials.

5. Pastoriza-Gallego, M.J., Casanova, C., Legido, J.L., \& Pioneiro, M.M., (2011). CuO in water nanofluid: influence of particle size and polydispersity on volumetric behavior and viscosity. Fluid Phase Equil. 300, 188 - 196.

6. Salinas, S. R. A. (2005). Einstein e a teoria do movimento browniano (Einstein and the theory of the Brownian movement). Revista Brasileira de Ensino da Física, v. 27, p. 263-269.

7. Timofeeva, E.V., Yu, W., France, D.M., Singh, D., \& Routbort, J.L., 2011. Nanofluids for heat transfer: an engineering approach. Nanoscale Res. Lett. 6, 182.

8. Yang, Y., Zhang, Z. G., Grulke, E. A., Anderson, W. B. \& Wu, G. (2005). Heat transfer properties of nanoparticle-in-fluid dispersions (nanofluids) in laminar flow. International Journal of Heat and Mass Transfer, 48, 1107-1116

9. Yu, W., France, D.M., Routbort, J.L., \& Choi, S.U.S., 2008. Review and comparison of nanofluid thermal conductivity and heat transfer enhancements. Heat Tran. Eng. 29, 432 - 460.

10. Zhu, D., Li, X., Wang, N., Wang, X., Gao, J., \& Li, H., 2009. Dispersion behavior and thermal conductivity characteristics of Al2O3 and $\mathrm{H} 2 \mathrm{O}$ nanofluids. Curr. Appl. Phys. 9, 131-139. 